\title{
An analysis of multi objective energy scheduling in PV-BESS system under prediction uncertainty
}

Raveendrannair, Unnikrishnan; Sandelic, Monika; Sangwongwanich, Ariya; Dragicevic, Tomislav; Costa Castello, Ramon; Blaabjerg, Frede

\author{
Published in: \\ IEEE Transactions on Energy Conversion
}

Link to article, DOI:

10.1109/TEC.2021.3055453

Publication date:

2021

Document Version

Peer reviewed version

Link back to DTU Orbit

Citation $(A P A)$ :

Raveendrannair, U., Sandelic, M., Sangwongwanich, A., Dragicevic, T., Costa Castello, R., \& Blaabjerg, F. (2021). An analysis of multi objective energy scheduling in PV-BESS system under prediction uncertainty. IEEE Transactions on Energy Conversion, 36(3), 2276 - 2286. https://doi.org/10.1109/TEC.2021.3055453

\section{General rights}

Copyright and moral rights for the publications made accessible in the public portal are retained by the authors and/or other copyright owners and it is a condition of accessing publications that users recognise and abide by the legal requirements associated with these rights.

- Users may download and print one copy of any publication from the public portal for the purpose of private study or research.

- You may not further distribute the material or use it for any profit-making activity or commercial gain

- You may freely distribute the URL identifying the publication in the public portal 


\title{
An analysis of multi objective energy scheduling in PV-BESS system under prediction uncertainty
}

\author{
Unnikrishnan Raveendran Nair, Monika Sandelic, Ariya Sangwongwanich, Tomislav Dragičević, Member, IEEE, \\ Ramon Costa-Castelló, Senior, IEEE, and Frede Blaabjerg, Fellow, IEEE
}

\begin{abstract}
Energy storage systems (ESSs) are being considered to overcome issues in modern grids, caused by increasing penetration of renewable generation. Nevertheless, integration of ESS should also be supplemented with an optimal energy management framework to ensure maximum benefits from ESS. Conventional energy management of battery, used with PV system, maximises self-consumption but does not mitigate grid congestion or address battery degradation. Model predictive control (MPC) can alleviate congestion, degradation while maximizing self-consumption. As such, studies will be carried out, in this work, to highlight the improvement with MPC based energy management over conventional method using simulations of one-year system behaviour. As MPC uses forecast information in decision making, the impact of forecast uncertainties will be assessed and addressing the same through constraint tightening will be presented. It is concluded that MPC provides improvement in system behaviour over multiple performance criteria.
\end{abstract}

Index Terms-Model predictive control, battery management, PV system, grid congestion degradation

\section{INTRODUCTION}

$\mathbf{T}$ HE increasing residential PV installations, especially in European grids, in the last decade have contributed to ever growing pool of cleaner energy sources. However, though they are environment friendly they may not be grid friendly. The reason being their intermittent generation and uncontrolled power injection to main grid leading to congestions, voltage regulation problems and instabilities [1], [2].

ESS can be utilised to overcome the above issues with PV integration but they need to be managed optimally [3]. The increased integration of PV sources have resulted in PV energy achieving grid parity [4]. This means that the owner (consumer) gets maximum economical benefit from utilising the generated PV energy in their premises (self-consumption) rather than sending it to the grid [4]. Herein lies the fundamental problem, as residential PV installation with battery energy storage system (BESS) uses the maximising self-consumption control strategy to achieve maximum economic benefit. This method charges the BESS whenever surplus PV power is

U.Raveendran Nair and R.Costa-Castelló are with Institut de Robòtica i Informòtica industrial, Universitat Politècnica de Catalunya, 08034 Barcelona Spain, (e-mail: uraveendran@iri.upc.edu, ramon.costa@upc.edu)

M.Sandelic, A.Sangwongwanich, T.Dragičević and F.Blaabjerg are with the Department of Energy Technology, Aalborg University, Aalborg 9220, Denmark.(e-mail: mon@et.aau.dk;ars@et.aau.dk; tdr@et.aau.dk ; fbl@et.aau.dk)

This work is done as part of project which has received funding from the European Union's Horizon 2020 research and innovation programme under the Marie Skłodowska Curie grant agreement No 675318 (INCITE). This work was supported in part by the Spanish Ministry of Economy and Competitiveness under Projects CICYT RTI2018-094665-B-I00, DOVELAR ref. RTI2018-096001-B-C32 (MCIU/AEI/FEDER, UE) and María de Maeztu Seal of Excellence to IRI (MDM-2016-0656), and by the Generalitat de Catalunya through the Project 2017 SGR 482.

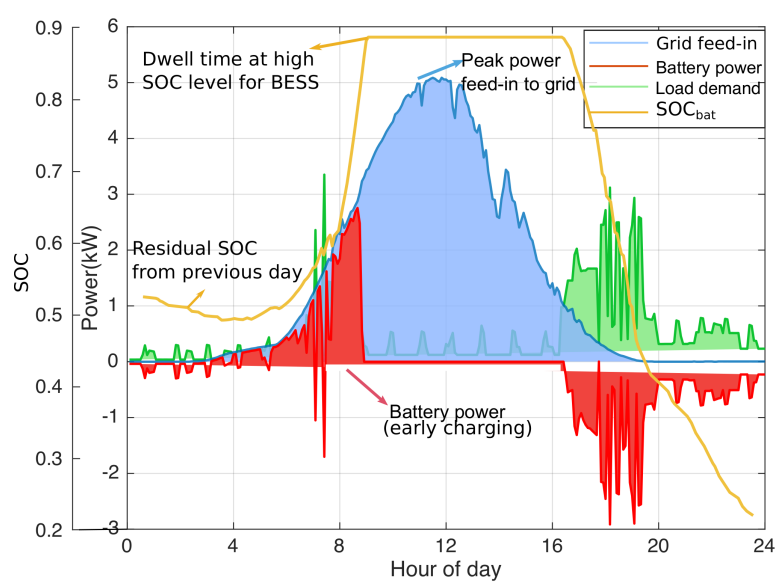

Fig. 1: Typical BESS charging, SOC and grid feed-in profile under maximising self-consumption strategy. Early full charging of BESS and subsequent peak power feed-in observed.

available as shown in Fig. 1 [5]. This leads to BESS being fully charged early in the day. Therefore, when peak PV generation occur the surplus power is fed to grid as in Fig.1. Typically, grid operator does not control this power injection from the consumers [2]. Irrespective of grid topology, if multiple PV units do the same without sufficient load demand the grid can be congested. This has been verified through multiple occurrences of such congestion events in European grids [2], [6], [7]. Nowadays, feed-in power limitations are imposed on PV systems to mitigate this [2], [4], [7] at the cost of PV power utilisation.

Another drawback with conventional maximising selfconsumption strategy is the BESS degradation. The two major ageing mechanism in BESS (Li-ion based) are the cyclic and calender ageing [8]. They are results of degradation arising from excessive cycling of BESS and increased dwell time at high SOC levels. In conventional scheme the early charging of BESS results in high SOC dwell periods (Fig.1) and increased BESS degradation [5], [8].

The grid congestion can be mitigated if the residential PV sources gradually inject power to grid rather than being concentrated at peak generation period. Utilising forecast information in individual BESS management can achieve this and have shown to limit these issues while ensuring good selfconsumption [9]. This knowledge of future generation and load profiles allow energy management (EM) systems to know the instance of peak PV generation, thus preventing early fullcharging of BESS (addressing calender ageing) and ensuring BESS capacity availability during peak PV generation. This limits the feed-in power to grid and congestion. The existing 
works using this approach of forecast information for EM of BESS have focussed on offline approaches [9], [10]. However, they result in sub-optimal system behaviour.

Online methods, like Model predictive control (MPC), can provide improved performance and are highly suited for these problems. The application of MPC for energy management in electric systems have been explored from the perspective of grid operator to improve operational efficiency [11], [12], operating cost [13], [14] and for energy arbitrage [15], [16]. Apart from economic aspects, MPC has also been applied for improving electric parameters, like voltage regulation [17], in grids having high penetration of renewable sources. The application of MPC has also focussed on centralised [13] [16] and distributed control [17], [18] strategies. However, an application of MPC to mitigate the practical issue of grid congestion (in many European grids) arising from the peak PV power injection from the consumer side installations have not been addressed. Another important aspect is the forecast uncertainty and how it affects MPC performance, which is not addressed by previous works [19]. Stochastic MPC techniques exist [20], however their application to practical problems have not been presented. This is relevant, as accounting for stochastic behaviour leads to conservative decision making by the MPC which significantly affect the economic benefit of using the same. A comprehensive, quantitative analysis regarding this, the deterioration of MPC performance under forecast errors and incorporation of real data from prediction systems to stochastic MPC techniques are missing in existing literature. Finally, the previous works do not present a complete MPC architecture integrating the prediction and decision making stage to demonstrate the practical implementation of MPC.

Considering the above, the main contributions of this work can be summarised as follows:

- Application of MPC for energy management in a PVBESS system at residential installations to address objectives like maximising self-consumption, minimising grid congestion and BESS degradation.

- Presenting a complete MPC framework integrating the prediction and decision making stages

- Addressing forecast uncertainty in MPC decision making using a simple, practically realisable constraint tightening approach based on real data from forecast stage while also ensuring that the decision making is not conservative.

- Quantitative analysis of MPC performance over conventional energy management method (maximising selfconsumption) and impact of forecast uncertainty on MPC.

In order to comprehensively present the quantitative analysis of MPC performance long term system behaviour (1 year) will be simulated and studied in this work. The rest of the paper is organised as follows. Section II introduces the predictive energy management scheme (PEMS) with the forecasting stage and MPC based scheduling. Section III presents the result of PV-BESS scheduling with the PEMS and quantifies the improvements achieved with the same. Finally the work is concluded in Section IV.

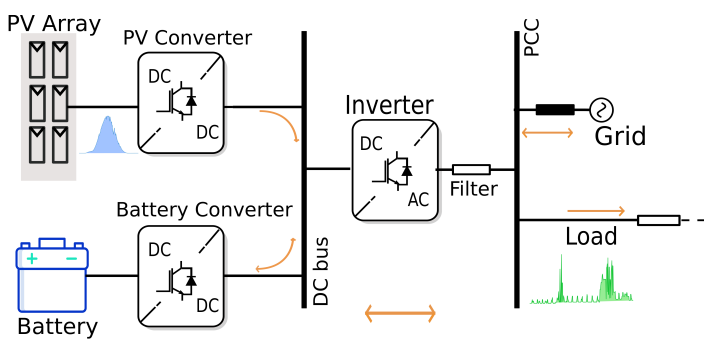

Fig. 2: Schematic of the test case microgrid.

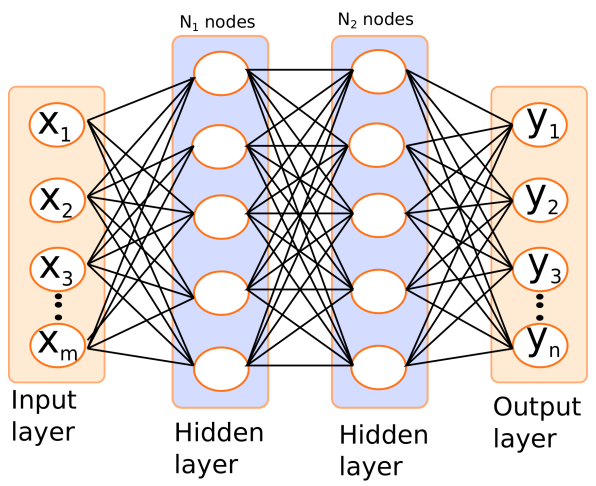

(a)

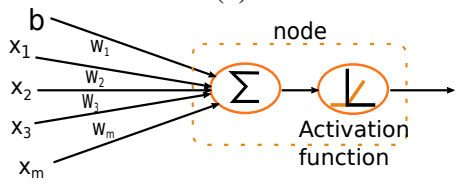

(b)

Fig. 3: (a) Feedforward neural network structure used in forecasting unit, (b) node representation.

\section{PREDICTIVE ENERGY MANAGEMENT SCHEME}

The PV-BESS system considered in this work is shown in Fig.2. The PEMS comprises of two stages, the forecasting unit and the MPC based scheduling unit.

\section{A. Forecasting unit}

A neural network (NN) [21] will be used as forecasting unit for the PEMS considered in this work. It should be noted that developing novel forecasting models as such is not a contribution of this work and hence NNs are considered. Despite NNs not being the most accurate forecast models [22], the predictions from $\mathrm{NN}$ have shown to provide a reasonable representation of actual system behaviour [23] and as such has been used in this work to demonstrate integration of forecast model with MPC.

The typical NN framework, used in this work, is shown in Fig. 3(a). It consists of an input layer, two hidden layers and an output layer. The two hidden layers have $N_{1}$ and $N_{2}$ nodes respectively. The structure of each node is shown in Fig. 3(b). The NN shown here is fully connected. The activation function at each node is a rectified linear unit ( ReLU) as shown in Fig. 3(b), chosen due to its better performance [24].

In order to obtain highly accurate predictions on generation or load profiles from the NN system, they need to be trained using the previous data of the same. The training process can be considered as a learning phase of the $\mathrm{NN}$, wherein the previous generation and load data is used by the $\mathrm{NN}$ to 
study the underlying behavioural pattern. The mathematical formulations involved in the training process and the related algorithms [25] are beyond the scope of this work. Interested readers are directed to [25] for the same.

\section{B. MPC based scheduling unit}

MPC is a modern optimal control strategy which enables handling of non-linearities and constraints efficiently [20]. In EM problems economic MPC is typically considered [20]. The MPC uses predicted value of generation, load profiles and an appropriate system model to predict evolution of system states into a future predefined time period called the control horizon. Using online optimization, MPC will modify the manipulated inputs so that the system states will follow an optimal trajectory in this horizon. This in-turn will guarantee that the system behaviour is optimised with respect to some predefined performance parameters.

At any sampling instant, $k$, for a control horizon $N$, the MPC generates $N$ set-points $\left(s_{0 \mid k}, s_{1 \mid k}, s_{2 \mid k} \ldots . . s_{N-1 \mid k}\right)$ where $s_{i \mid k}=s(k+i) \quad \forall i=0,1,2 \ldots, N-1$. The first set-point, $s_{0 \mid k}$, will then be applied to the system. This process is repeated at every sampling instant using the sampled value of system states at that moment. This ensures that the control action is generated using latest system states, thus giving a sense of feedback [20], [26]. The formulation of the optimisation problem used in MPC for EM in PV-BESS system is discussed next.

1) Cost function: The cost function

$$
J(k)=\min \sum_{i=k}^{k+N-1}\left(J_{\text {grid }}(i)+J_{\text {bess }}(i)\right),
$$

defines the aspects of PV-BESS system operation that are being optimised. There are two parts to the above cost function. The first part $J_{\text {grid }}$ is defined as

$$
J_{\text {grid }}(i)=\lambda_{g} \cdot p_{\text {grid }}(i)^{2}
$$

where $\lambda_{g}$ is a penalising weight. The above cost term penalises the power interaction with the grid $\left(p_{\text {grid }}\right)$. As a result, if (1) is to be minimised the EM scheme will try to keep the values of $p_{\text {grid }}$ as low as possible. This will ensure that grid feed-in is reduced. By minimising grid feed-in, the MPC indirectly forces the generated PV power to be utilised at the consumer premises, as far as possible. This increases self-consumption.

The second term in (1), is given by

$$
J_{\text {bess }}(i)=\lambda_{s} \cdot S O C(i)^{2}+\lambda_{d} \cdot \Delta S O C(i)^{2}
$$

minimises battery degradation. $\lambda_{s}, \lambda_{d}$ are weighting factors. As mentioned before, in Li-ion batteries, the calender, cycling ageing are accelerated through high SOC dwell times and excessive BESS cycling respectively [8]. These effects are minimised through (3). In (3) high SOC values are penalised, thereby minimising dwell times at highly charged states for BESS. The $\triangle S O C$ in (3) penalises change in SOC and thereby excessive cycling. The explicit equation defining the battery degradation is not considered here, as it is non-linear [5]. This can lead to increased complexity in solving the optimisation problem [27]. The quadratic functions, shown above provides a reasonable approximation which can be efficiently solved.
It should be noted that the above cost function does not limit power interaction with grid or utilisation of BESS if penalising weights are chosen appropriately. The (2) along with the first term in (3) provides a temporal shift of BESS charging to peak generation period. This reduces the dwell time of BESS at high SOC and peak power injection to grid. It should be noted that the $\lambda_{d}$ in (3) should be chosen judiciously to prevent under utilisation of BESS capacity. This aspect will be discussed in subsequent sections.

2) BESS model: MPC used ESS and grid models during the optimization. The BESS model is based on the Coulomb counting equation given by [28]

$$
S O C(i+1)=S O C(i)-\frac{T_{s}}{C_{b e s s}} \cdot p_{b e s s}(i)
$$

where $T_{s}$ is the sample period, $p_{\text {bess }}(i)$ is the power set point and $C_{\text {bess }}$ is the capacity of BESS.

3) Grid model: The grid is modelled using the power balance equation as follows:

$$
p_{\text {bess }}(i)+p_{\text {gen }}(i)+p_{\text {grid }}(i)-p_{\text {load }}(i)=0
$$

where $p_{\text {grid }}(i)$ is the power exchanged with the grid, $p_{\text {gen }}(i), p_{\text {load }}(i)$ are the power generated by the RES and load demand respectively at time instant $i$.

4) Constraints: The problem constraints address physical limits of ESS and power limits of the power converter. These constraints are ensured by

$$
p_{\mathrm{c}}^{\min } \leq p_{\text {bess }}(i) \leq p_{\mathrm{c}}^{\max }
$$

where $p_{c}^{\min }$ and $p_{c}^{\max }$ are maximum power handling limits of the bidirectional converters of BESS.

The physical limits of BESS are addressed through [29]

$$
S O C^{l} \leq S O C(i) \leq S O C^{u}
$$

where $S O C^{l}$ is the lower bound and $S O C^{u}$ is the upper bound on SOC of BESS. These bounds also protect from degrading stress in BESS arising from high charged or deep discharged states [29]. Introducing hard constraints like (7) can cause non-convergence of the optimisation problem. This can be overcome by using soft constraints [30]. The soft constraints allow some violation in the bounds on SOC, but improves the convergence of the optimisation problem. The (7) represented as soft constraints is given by

$$
S O C^{l}-\epsilon_{\text {bess }} \leq S O C(i) \leq S O C^{u}+\epsilon_{\text {bess }}
$$

where $\epsilon_{\text {bess }}$ is the slack variable which indicates the constraint violation. In order to ensure that the SOC limits are not significantly violated, the slack variables are penalised using a cost function like

$$
J_{\text {soft }}=\lambda_{\epsilon} \cdot \epsilon_{\text {bess }}^{2} .
$$

Therefore, the final optimisation problem used for set-point generation by MPC is summed up as

$$
\min \sum_{i=0}^{N-1}\left(J_{\operatorname{grid}(i \mid k)}+J_{\text {bat }(i \mid k)}+J_{\operatorname{soft}(i \mid k)}\right)
$$


subject to

$$
\text { ESS and Grid models (4), (5) }
$$$$
\text { Constraints (8), (6). }
$$

The above represents a quadratic programming problem which can easily be solved with solvers like Gurobi [31].

The optimal behaviour with predictive management unit relies on the accuracy of the forecast. A proper training of NN based forecasting unit can reduce, but not eradicate it. Therefore to account the forecast errors, the optimisation problem in MPC needs modification. In this context, a simple constraint tightening approach can ensure this [32].

In PV-BESS system, the critical information that the MPC needs from forecast is the total imbalance energy in a control window and the instance of peak imbalance power (peak generation). This enables decision on the degree of utilisation of BESS and when to deploy BESS charging/discharging to prevent grid congestion. The forecasting unit can give a fairly accurate indication of the peak generation periods (highlighted in the next section). However, errors exist in the predicted total imbalance energy in a control window.

The forecast at any instant is not definitive but vary within a certain bound $d_{i \mid k}$ defined as

$$
d_{i \mid k}= \begin{cases}0, & \text { for } i=0 \\ {\left[+\Delta \theta_{i},-\Delta \theta_{i}\right] .} & \text { for } i=1,2 . . N-1 .\end{cases}
$$

At the sampling instant $k$ the actual value is known from sampled data, hence $d=0$ at $i=0$. As for the other points in the control horizon, $d_{i \mid k}$ should be accounted by MPC in its decision making. In constraint tightening, this is achieved by modifying the bounds of the constraints, (11), in the optimisation problem. In BESS this will modify the SOC limits, thus ensuring that there is always some buffer capacity to accommodate for the prediction error. Defining $p_{\text {grid (i } \mid \mathrm{k})}-p_{\text {load(i|k) }}$ as $p_{\text {def(i|k) }}$ (predicted deficient power in the system), (5) is rewritten under prediction uncertainty as

$$
p_{\text {bess }(i \mid k)}+p_{\operatorname{gen}(i \mid k)}+u_{(i \mid k)}+p_{\operatorname{def}(i \mid k)}+d_{(i \mid k)}=0 \text {. }
$$

In the above equation additional term $u_{i \mid k}$ is the control action from the low-level controllers to counteract the uncertainty in forecast $d_{(i \mid k)}$. The predictive management unit bases its decision on the forecast value $p_{\operatorname{def}(\mathrm{i} \mid \mathrm{k})}$ which is the deterministic part of the imbalance forecast. Therefore (5) is split into the deterministic part catered by the PEMS

$$
p_{\text {bess }(i \mid k)}+p_{\operatorname{gen}(i \mid k)}+p_{\operatorname{def}(i \mid k)}=0
$$

and the uncertain part catered by low-level controller.

$$
u_{(i \mid k)}=-d_{(i \mid k)} \text {. }
$$

This requires that the bounds (8) and (6) are modified to ensure sufficient margin is available for the low-level control to counteract $d_{(i \mid k)}$. The power bounds, (6), are modified using (15) as

$$
p_{\text {bess }}^{\min }+u_{(i \mid k)} \leq p_{\text {bess }(i \mid k)} \leq p_{\text {bess }}^{\max }-u_{(i \mid k)} .
$$

leaving some margin for the low-level controller to modify the power setpoints from predictive management unit to account for $d_{(i \mid k)}$ without exceeding power limits.
In order to modify the SOC bounds (8) the BESS model (4) is represented using $u_{i \mid k}$ under uncertainty in forecast as

$$
\begin{array}{r}
S O C(i+1 \mid k)=S O C(i \mid k)- \\
\frac{T_{s}}{C_{b e s s}} \cdot\left(p_{\text {bess }(i \mid k)}+u_{(i \mid k)}\right)
\end{array}
$$

under assumption that $d_{(i \mid k)}$ is always catered by BESS until fully charged/discharged. This ensures maximum selfconsumption as well. The above is rewritten in terms of sampled $S O C$ value at instant $k\left(S O C_{\mathrm{bess}(0 \mid k)}\right)$ as

$$
\begin{aligned}
& S O C(i+1 \mid k)=S O C(0 \mid k)- \\
& \frac{T_{s}}{C_{\text {bess }}} \cdot \sum_{l=0}^{i}\left(p_{\text {bess }(l \mid k)}+u_{(l \mid k)}\right) .
\end{aligned}
$$

The deterministic part in the above equation is

$$
S O C(i+1 \mid k)=S O C(0 \mid k)-\frac{T_{s}}{C_{\text {bess }}} \cdot \sum_{l=0}^{i}\left(p_{\text {bess }(l \mid k)}\right)
$$

and

$$
\frac{T_{s}}{C_{b e s s}} \cdot \sum_{l=0}^{i} u_{(l \mid k)}=d x_{(i \mid k)}
$$

forms the non deterministic part. Based on this, constraint tightening of (8) is done as follows

$$
\begin{array}{r}
S O C^{l}-\epsilon_{\mathrm{bess}}+d x_{(i \mid k)} \leq S O C(i) \\
\leq S O C^{u}+\epsilon_{\mathrm{bess}}-d x_{(i \mid k)}
\end{array}
$$

Finally the optimisation problem can be reformulated as

$$
\min \sum_{i=0}^{N-1}\left(J_{\operatorname{grid}(i \mid k)}+J_{\text {bat }(i \mid k)}+J_{\operatorname{soft}(i \mid k)}\right)
$$

subject to

$$
\begin{array}{r}
\text { ESS and Grid models (19),(14) } \\
\text { Constraints (21), (16) }
\end{array}
$$

Another important factor in defining the optimality of the solution is the size of $\Delta \theta$. A higher value of the same causes higher tightening of constraints and more conservative decision making. An increased tightening prevents BESS from charging or discharging too much, leading to low selfconsumption, as the PV power is being sent to grid. In a site level system, like the PV-BESS of this work, the intraday variations in the load/generation profile tends to be high in comparison to an aggregated system. Forecasting the behaviour of such systems is always a tough problem as shown in [33]. In this scenario, defining $\Delta \theta_{i}$ based on a mean average absolute error for every point in a prediction horizon leads to larger bounds $d_{(i \mid k)}$. However, if the error is aggregated over a forecast window there will be cancellation of error along the prediction window. This can result in a less conservative definition of error bounds if this mean absolute aggregate error, $e_{a}$, (for a prediction window) is taken to define $d_{(i \mid k)}$.

The distribution of $e_{a}$ along $d_{(i \mid k)}$ can be done based on behaviour of forecasting unit. In any prediction window the initial values are highly correlated with the previous values compared to latter values. As such the errors in forecast of 


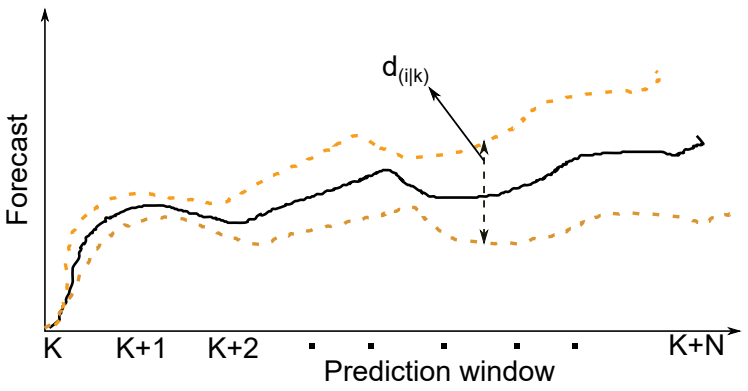

Fig. 4: Depiction of error propagation along forecast window.

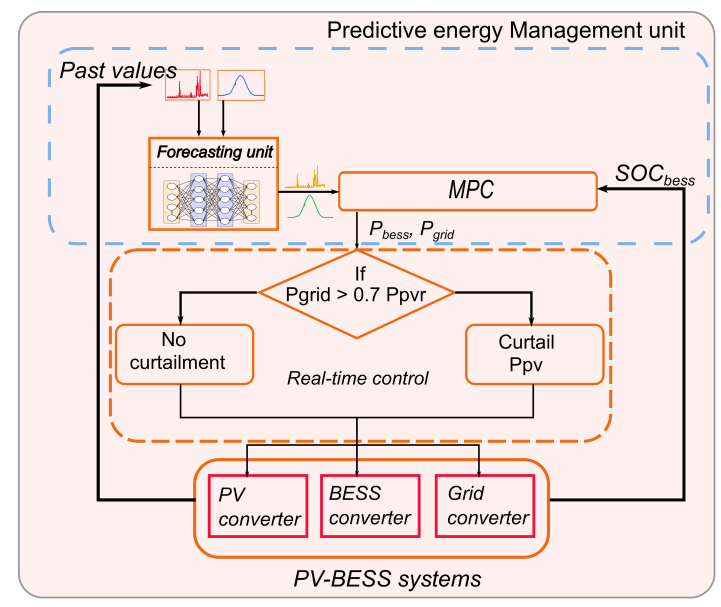

Fig. 5: Flowchart for the energy management decision making process, using predictive control, for PV-BESS system.

initial values will be lower. Therefore, distribution of $e_{a}$ in an increasing manner along a prediction horizon, as shown in Fig.4, to define $d_{(i \mid k)}$ makes practical sense.

\section{Grid feed in limitation}

To mitigate grid congestion from high surplus PV power feed-in, restriction are imposed by utility operators. An example, is the case in Germany where PV systems with a power rating less than $30 \mathrm{~kW}$ have to limit their feed in at $70 \%$ of their nominal value [34]. This condition can be easily implemented with MPC through constraints on $p_{\text {grid }}$. However, to ensure recursive feasibility an additional term will be introduced in (14) to define the power curtailment. Adding additional decision variables in the optimisation problem will require that the penalising weights for them be defined optimally. Failure to define the same can result in sub-optimal result. Apart from this, the additional decision variable for power curtailment will increase the computational demand of MPC. Therefore to overcome this issue and provide a solution that can be implemented practically with minimal computational resources, the optimisation problem will be solved as in (22) with (23) and the low-level controllers will ensure the power curtailment.

The entire PEMS for the PV-BESS with real-time control unit, handling power curtailment, is shown in Fig. 5. This presents a hierarchical control scheme.

\section{RESULTS AND DISCUSSION}

The PV generation and load demand are emulated using one year's data from a test microgrid in Lindenberg, Germany
[7]. The load represents a four person household with annual demand of 4.5 MWh. The data was obtained using a sampling time of 5 minutes resulting in 288 samples a day. The sizing of the BESS is chosen based on the results of the works [4], [35] which proposes 1-1.55 kWh BESS capacity per $1 \mathrm{~kW}$ of installed PV capacity. Considering this an optimal battery sizing of $9.375 \mathrm{kWh}$ for $6 \mathrm{~kW}$ of PV array power was chosen for this work. The above represents an optimal BESS sizing considering cost of investment and BESS degradation. An in depth discussion on the same can be found in above references.

In this work, considering the daily cyclic behaviour of the PV-BESS system, the control horizon in MPC (N) was chosen as 24 hours. Therefore, objective of the forecasting unit at any instant will be to predict the next 24 hours.

It should be noted that the BESS model (7) had been represented as a linear approximation using coulomb counting model. This was done with the view of keeping the optimisation problem quadratic. Considering the long prediction horizon of 24 hours and 5 minute sampling interval, the decision variables in the optimisation problem is close to 600. In this case using more complex non-linear representation of BESS may not guarantee convergence of the optimisation problem to solution. Apart from this given the smaller sampling time of 5 minute, linear representation of (7) gives a fairly accurate representation of BESS behaviour.

\section{A. Forecasting unit results}

The NN was realised in Python using the Keras package [36]. Training was done with the ADAM solver [37]. In order to avoid overfitting of data cross validation and early stopping [38] was ensured during the NN training. One year's data was available of which $70 \%$ was used for training and remaining for testing in both PV and load forecast.

The NN input, used in PV forecast, was the last 72 hours of actual generation data based on the correlation of the PV generation to previous values. Explicit weather data was not available and hence was not used as input to NN. This resulted in a NN with input layer size of 864. The hidden layer size, $N_{1}$ and $N_{2}$, was chosen as 300 through multiple trials. The hidden layer size selection as shown here is a manual search method and may not be optimal. More optimal methods can be used in the selection of the same, nevertheless since the scope of the work is the energy management framework for a PV-BESS system they where not investigated in detail here. The PV generation forecast with $\mathrm{NN}$ for a 24 hour period is provided in Fig.6. The forecast shown here is during autumn where the solar irradiation tends to be lower. The accuracy of the prediction can further be improved using weather data which was not available in this case. Nevertheless, it should be noted that the PV forecast is able to predict the time period where high generation occurs which is vital for the PEMS decision making.

The NN for the load forecasting used the last 168 hours (1 week) measured load demand as input. Unlike PV forecast, the one week data was used since the load demand on any day had high correlation with last weeks load demand on same day (identified through analysis of correlation plots of available data). This resulted in an input layer size of $2016(288 \cdot 7)$ 


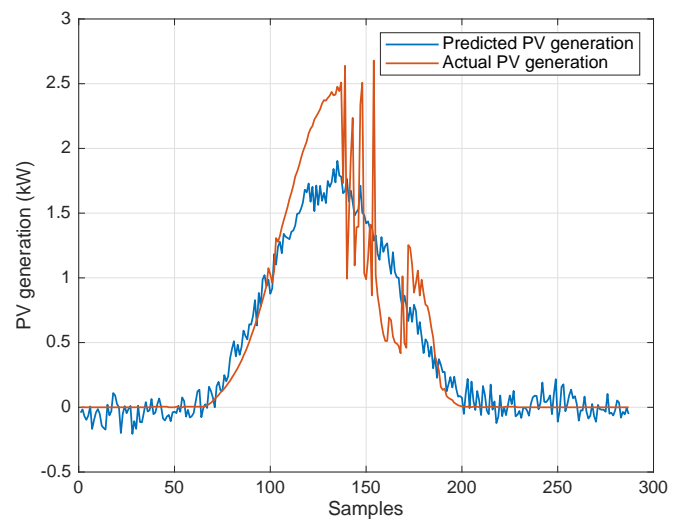

Fig. 6: Result of one day's PV generation forecast using NN on testing data.The data is from 12 October 2004.

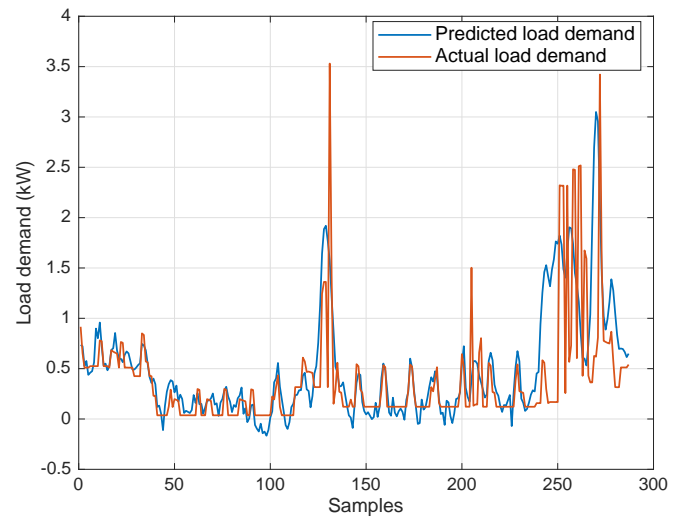

Fig. 7: Result of load demand forecast using NN.

for NN forecasting load demand. The size of $N_{1}$ and $N_{2}$ was chosen as 300 through multiple trials. The results using the same for load forecast for a 24 hours period is shown in Fig. 7.

The PV and load forecast results highlight the difficulty in site level prediction (small systems), as discussed before. As shown in Fig.6 and Fig.7, the real data has sudden variations and the forecasting units could not entirely reproduce them. This justifies the importance of using mean aggregate absolute error to define $d_{(i \mid k)}$ as discussed before. The aggregation of error will reveal the difference in total energy between predicted and forecast value in a prediction window. For example, in the case of PV forecast, the aggregation of errors will provide an indication of total generated energy difference between real and forecast value, in a prediction window. The same is the case with load forecast. As a result, this mean absolute aggregate error is also analysed here. In order to get the mean aggregate absolute error, the absolute value of aggregated error for all prediction windows of the year (based on actual data) was calculated and the mean of this value was used. Analysing the actual PV generation data, it was observed that the mean aggregated energy of PV system in a moving 24 hour prediction window was $13.56 \mathrm{kWh}$. Based on the forecast data the mean absolute aggregate error in the same moving prediction window was $3.28 \mathrm{kWh}$. This accounted to $23 \%$ the average energy value for a prediction window. Similarly, for
TABLE I: System parameters and their values used for the energy management strategy.

\begin{tabular}{|l|l|}
\hline Parameter & Value \\
\hline Rated power of PV system $P_{\text {pvr }}$ & $6 \mathrm{~kW}$ \\
\hline$C_{\text {bess }}$ & $9.375 \mathrm{kWh}$ \\
\hline Sampling time & 5 min \\
\hline MPC horizon (N) & 24 hours \\
\hline$p_{c}^{\text {min }}, p_{c}^{\text {max }}$ & $3 k W$ \\
\hline$S O C^{u}, S O H_{b e s s}^{l}$ & $0.9,0.1$ \\
\hline$\lambda_{g}, \lambda_{s}, \lambda_{d}$ & $500,200,3$ \\
\hline
\end{tabular}

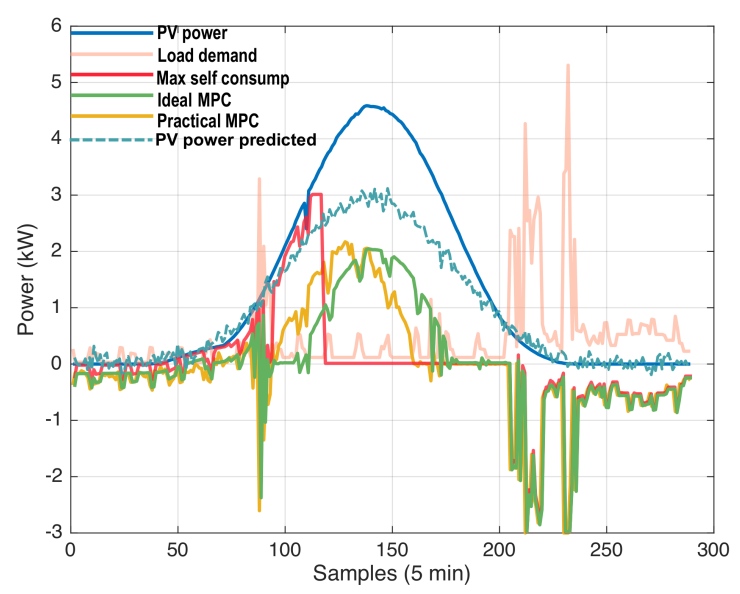

Fig. 8: Typical daily BESS power profiles with the different energy management strategies The early full charging of BESS with the conventional method is overcome with PEMS.

the load, the mean aggregate energy demand of the load in a moving prediction window was $10.81 \mathrm{kWh}$, whereas the mean aggregate absolute error for the same was $1.015 \mathrm{kWh}$. This represented an error, that was $9.3 \%$ the average energy value. In comparison to load data the aggregated error in PV prediction is higher. This is mainly due to the limited amount of data available for the ANN training (only 1 year data was used) and the lack of weather data as additional input feature.

The power deficiency profile $\left(p_{\operatorname{def}(i \mid k)}\right)$, used in MPC, was obtained from PV and load forecast. The individual aggregated error of PV and load forecast was then used to determine $e_{a}$.

\section{B. Predictive energy management}

The EM in the PV-BESS system will be carried for one year. The results from annual scheduling enables quantification of the long-term improvements that can be achieved with PEMS. The parameters used in the optimisation of the PEMS is shown in Table. I. Prior to presenting the results some performance measures will be introduced. An important measure that will be analysed is the self-consumption ratio (SCR). SCR defines the percentage of total PV generation used by the microgrid system to meet its load demand (including energy storage in BESS). This is defined as [5]

$$
S C R=\frac{E P V_{\text {consumed }}}{E P V_{\text {generated }}} \cdot 100(\%)
$$

where $E P V_{\text {consumed }}$ is the annual PV energy utilised by the consumer and $E P V_{\text {generated }}$ is the total annual PV energy 

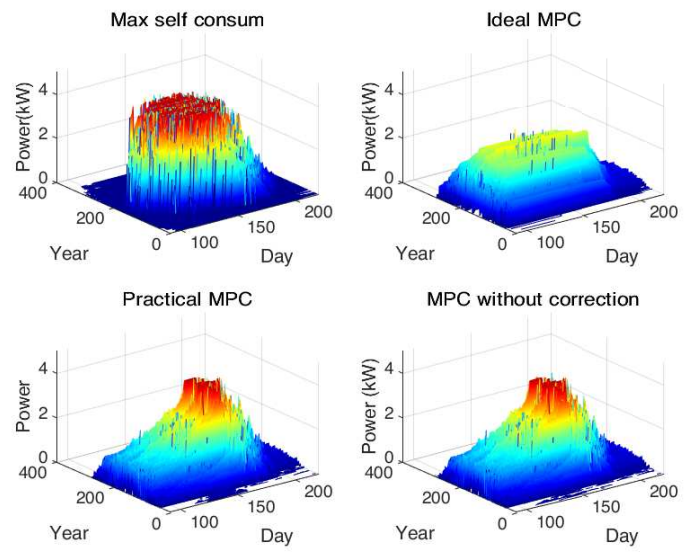

Fig. 9: Grid power feed-in profiles with different energy management schemes.

generated. Another measure of interest will be the annual BESS degradation resulting from the EM strategy. The BESS profile generated from the EM strategy will be used in a BESS degradation model defined in [5] to assess the degradation.

The penalising weights $\lambda_{g}, \lambda_{s}, \lambda_{d}$, as shown in Table I (not normalised), was chosen to get maximum SCR. A parameter sweep for different values $\lambda_{g}, \lambda_{s}, \lambda_{d}$ was carried to identify the optimal values shown in Table I. It should be noted that $\lambda_{d}$ value is low compared to others. A higher value for $\lambda_{d}$ will lead to small cycling of BESS which in-turn leads to underutilisation of BESS capacity. Therefore, as a trade-off a lower value was chosen. This can result in cycling ageing not being significantly reduced, however the higher value of $\lambda_{s}$ will ensure that the calender ageing is minimised.

The Fig. 8 shows BESS charging profile in a 24 hours period with different strategies namely: the conventional maximising self-consumption method, an ideal predictive management (ideal MPC) where perfect forecast of generation, load profile exists and the PEMS with constraint tightening (Practical MPC). Unlike conventional method, in PEMS, BESS does not undergo early charging as shown in Fig.8. The charging of the BESS is shifted to period of higher generation. Comparing the ideal and practical MPC performance, it can be observed that the BESS charging profile is shifted to an earlier instance when uncertainty exist. In the result shown in Fig.8, the predicted generation was lower than that of the actual generation. This forced the practical PEMS to charge the BESS earlier than in the ideal case. Despite this the BESS charging was closer to the peak generation period in the practical case, in comparison to the conventional scheduling strategy.

The grid feed-in profiles are shown in Fig.9. The results shown here is after grid power feed-in curtailment (70\% of the nominal value). In the conventional maximising selfconsumption method the early BESS charging results in peak power injection to grid as expected. The feed-in curtailment minimises the impact of this injection but at the cost of lesser utilisation RES generation. In the ideal MPC, as shown in Fig.9 this is completely mitigated with the prefect knowledge of future generation and load demand. In the case of practical MPC, the BESS reached full charge earlier than ideal MPC

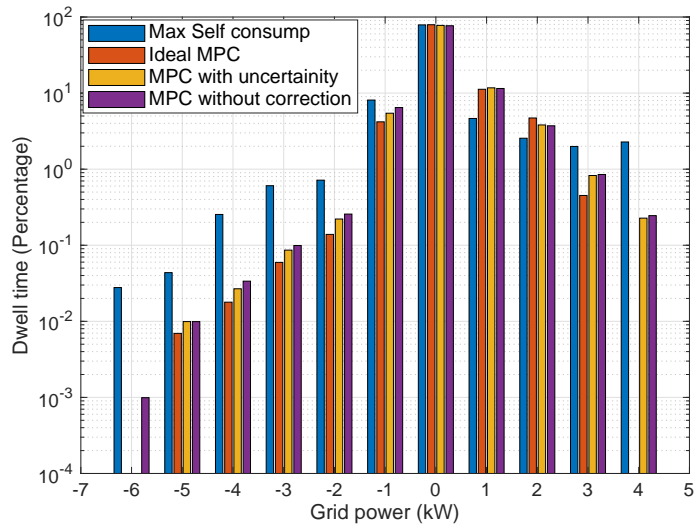

Fig. 10: Dwell times of power levels exchanged with the main grid under the different energy management schemes.

TABLE II: Comparison of SCR, BESS degradation and annual power curtailment for the different scheduling methods.

\begin{tabular}{|l|l|l|l|}
\hline $\begin{array}{l}\text { Scheduling } \\
\text { method }\end{array}$ & $\begin{array}{l}\text { Annual } \\
\text { SCR (\%) }\end{array}$ & $\begin{array}{l}\text { Annual BESS } \\
\text { degradation (\%) }\end{array}$ & $\begin{array}{l}\text { Annual PV power } \\
\text { curtailed (kW) }\end{array}$ \\
\hline $\begin{array}{l}\text { Conventional } \\
\text { method }\end{array}$ & 54.6 & 3.94 & $542.5 \mathrm{~kW}$ \\
\hline Ideal MPC & 54.3 & 3.71 & 0 \\
\hline Practical MPC & 52.71 & 3.73 & $35.91 \mathrm{~kW}$ \\
\hline $\begin{array}{l}\text { MPC without } \\
\text { correction }\end{array}$ & 52.73 & 3.65 & $37.83 \mathrm{~kW}$ \\
\hline
\end{tabular}

as shown in Fig.8. This leads to a small period of peak power injection to grid as shown in Fig.9. It should be noted that even with forecast errors, the time duration of peak power injection in constraint tightened MPC is significantly smaller than the conventional method

This is further highlighted in Fig.10 which shows the dwell times at different grid feed-in power levels. The PEMS with uncertainty in prediction shows lower dwell time at high feed-in power levels $(>3 \mathrm{~kW})$ compared to conventional maximising self-consumption scheme.

1) Self-consumption ratio assessment: The assessment of PV power self-consumption is shown in Table II, where annual SCR with different scheduling methods are compared. Considering a particular BESS sizing, the maximum SCR of $54.6 \%$ was observed in conventional maximising selfconsumption method. The SCR with ideal MPC is $54.32 \%$ which is $99.5 \%$ of the conventional scheme. The slight drop in self-consumption is attributed to the EM objective of lower BESS degradation. The behaviour of SCR and BESS degradation tend to be complementary. In the practical scenario SCR falls lower than the ideal case. This is expected due to the errors in prediction, which results in some usable power from the PV generation not utilised and being send to the grid. Nevertheless, it should be noted that the SCR in the practical scenario is still not very low in comparison to the conventional scheme at $52.71 \%$, which is $96.5 \%$ the maximum possible value. This value was achieved despite higher error in PV prediction due to absence of weather data. The availability of this data will only improve the prediction accuracy and improve SCR. This highlights the economic 


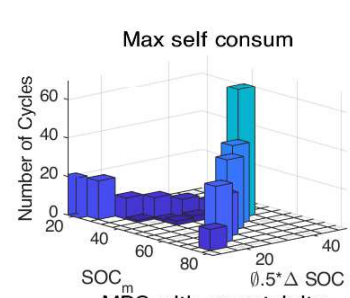

MPC with uncertainity
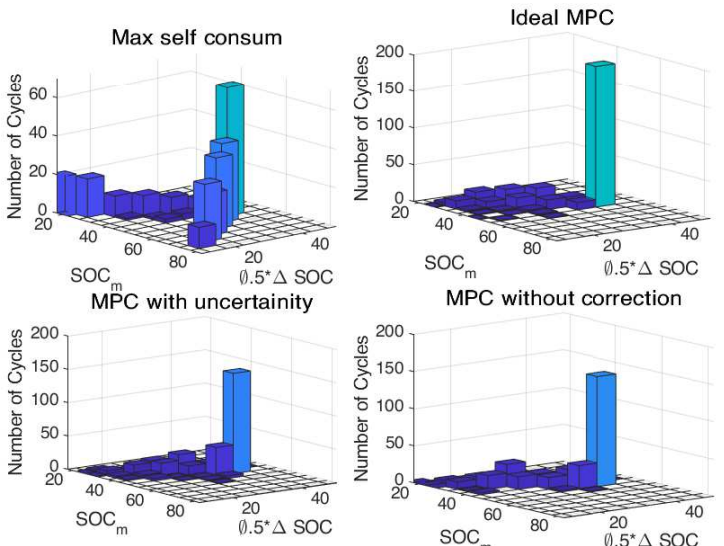

MPC without correction

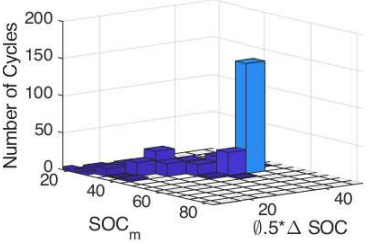

Fig. 11: BESS cycling undergone with different energy management schemes.

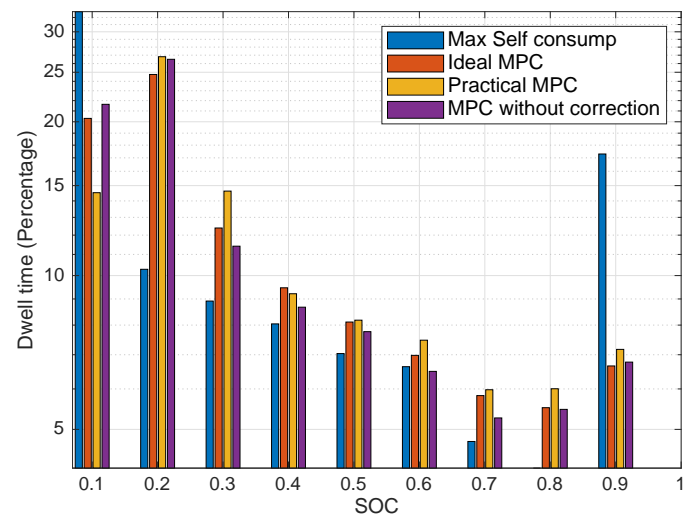

Fig. 12: Percentage dwell at various SOC levels by BESS with different schemes.

suitability of predictive management in practical scenarios where uncertainty in forecast exists.

2) Feed-in power limitation assessment: The Table II also shows the annual PV power curtailment with each management scheme. The conventional method, due to early battery charging, has maximum annual power curtailment of 542.5 $\mathrm{kW}$. In contrast the ideal MPC, with perfect forecast, manages PV-BESS operation with zero curtailment. In event of forecast error, as shown in Fig.9 peak power feed-in occurs and as such the need for curtailment. Nevertheless, as shown in Table II the PV power curtailed is only $34.91 \mathrm{~kW}$ which is almost $94 \%$ lesser than conventional method. This highlights increased utilisation of PV power with PEMS.

3) Battery degradation assessment: The BESS degradation with different strategies is shown in Table II. The degradation can be better explained with Fig.11, Fig.12 which shows the cycling and SOC dwell times of the BESS respectively. The plot in Fig.11 was generated from BESS SOC profile using rainflow algorithm. The $\mathrm{x}$-axis of Fig. 11 indicate half of the cycle magnitude whereas the y-axis indicates the mean SOC value of a cycle. For example, if BESS cycles between 10-90\% SOC, the $\mathrm{x}$ and $\mathrm{y}$-axis values will be 45 .

The conventional strategy exhibits the highest BESS degradation while predictive strategies (ideal and practical case) show almost a $6 \%$ reduction in annual BESS degradation. The early BESS charging in the conventional scheme results in increased dwell times at high SOC levels as shown in

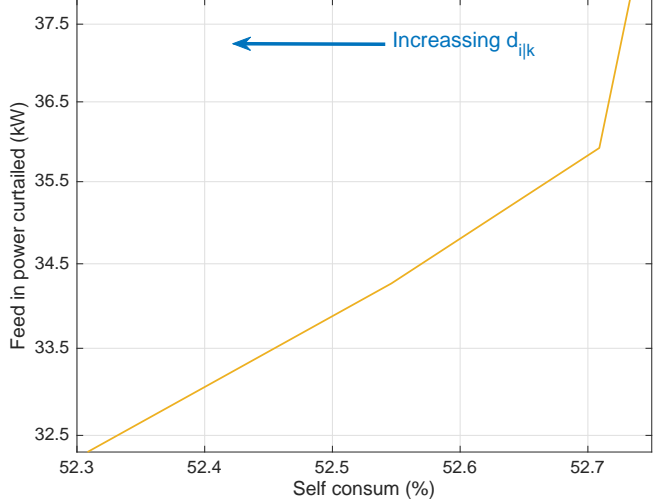

Fig. 13: Variation of feed in curtailment and self-consumption with increasing uncertainty bound $\left(d_{i \mid k}\right)$ on forecast values.

Fig.12, causing calender ageing. However, high SOC dwell times and calender ageing is significantly reduced in predictive strategies, due to shifting of the BESS charging to peak generation period. The predictive strategies however exhibit higher cycling (Fig.11) and therefore should have higher cycling ageing in comparison to conventional scheme. Nevertheless, the improvement in calender ageing outweighs this effect.

The annual degradation in BESS for the ideal and practical case is very similar. Assessing the cycling plot in Fig.11 it can be observed that the ideal MPC case undergoes a higher number of large magnitude cycles in comparison to the practical case. In comparison, the practical case has larger dwell times at high SOC levels due to earlier charging of BESS compared to ideal case. Therefore the combined effect of the both leads to similar degradation in both cases of PEMS.

It can be concluded that with the PEMS the selfconsumption (3.5\% in worst case) is slightly lesser that the conventional method. However, it is a small cost for benefits in reduced degradation of BESS, power curtailment and peak power feed-in. An exact assessment of the monetary benefit achieved by the reduction in BESS degradation in comparison to loss of self consumption cannot be accurately assessed with one ear simulation. This require simulation till end of BESS lifetime and calculation of levelized cost of energies as shown in [5], [39]. Such an in-depth economic analysis is beyond the scope of this work at present. Apart from this, the lack of sufficient data from the test microgrid makes it impossible to carry out long terms simulation needed to study the behaviour of BESS till end of lifetime.

Finally, an analysis of constraint tightening's effect on the objectives considered in the PEMS is carried out. The performance of PEMS without the tightening (but with imperfect forecast) is shown in Fig.9,10, 11, 12, as MPC without correction. Based on Fig.10, the grid feed-in performance for MPC with and without constraint tightening appears similar. However, assessing Table II it can be seen that, without constraint tightening, the annual grid feed-in curtailment tend to be higher whereas the SCR tends to be better. This is due to the conservative nature of decision making of PEMS due to constraint tightening. The PEMS expects a deviation in forecast value and as such prevents the BESS from charging or 
discharging excessively. This leads to some usable PV power being send to grid, thus lowering SCR. This also results in BESS from not being fully charged at peak generation time thereby limiting grid feed-in curtailment. Therefore increasing the bounds on uncertainty and the resulting increase in constraint tightening always reduces the feed-in curtailment but at the cost of lower SCR as shown in Fig.13. The annual BESS degradation is also lesser without constraint tightening as shown in Table.II. This is also a result of the conservative decision making of PEMS, which results in the BESS having a increased dwell times at higher SOC levels as evident from Fig.12. These results also justify the use of mean absolute aggregated error in the prediction window for defining the bounds $d_{i \mid k}$ instead of utilising the mean aggregated error at every point in the prediction window. This is because utilising the point wise error would have resulted in higher bounds in $d_{i \mid k}$ (considering the bigger error in PV forecast) leading to very conservative decision making.

\section{CONClusion}

The paper has proposed a practical framework for predictive energy management of residential PV-BESS installations comprising of a forecasting and MPC based scheduling stage. The proposed system have shown improved performance over the conventional maximising self-consumption control based on the results discussed above. The MPC based system with perfect forecast achieved almost $99.5 \%$ self-consumption (SCR) compared to conventional method while achieving 6\% reduction in BESS degradation without any power curtailment and significantly improved grid feed-in profile to mitigate congestion. This highlights improvement in multiple domains with MPC over conventional method. Even with uncertainty in forecast, annually, the MPC achieved $96.5 \%$ SCR with $6 \%$ reduction in BESS degradation, close to $94 \%$ reduction in power curtailment and improved grid feed-in profile compared to conventional method. This loss in SCR is expected as the MPC is a method that utilises forecast in its decision making and the error in forecast will affect its performance. The most important aspect to be ensured is that the deterioration in performance is not high enough that the utilisation of MPC cannot be justified. This was achieved and demonstrated here by making less conservative decisions with MPC through characterising the forecast error using the mean aggregated absolute error in a prediction window.

It should also be noted that the results achieved were with NN based forecast model which is not necessarily highly accurate. The performance of the energy management system can be further improved using more accurate forecast models, which can be a basis of future work. As discussed before, the penalising weights were determined through a parameter sweep. An aspect for future work can be using more analytical methods for optimal weight determination unlike heuristic methods employed in this work. It should also be noted that an in depth cost benefit analysis was not carried out here which require assessment till end of BESS lifetime. An aspect for future work can be a comprehensive economic analysis of different energy management strategies employed in PV-BESS system. Finally, future work can also focus on distributed
MPC control strategies to improve the power injection to grid by accounting for behaviour of other consumers in the grid infrastructure.

\section{REFERENCES}

[1] H. Schermeyer, M. Studer, M. Ruppert, and W. Fichtner, "Understanding distribution grid congestion caused by electricity generation from renewables," in Smart Energy Research. At the Crossroads of Engineering, Economics, and Computer Science. Springer, 2017, pp. 78-89.

[2] B. Bayer, P. Matschoss, H. Thomas, and A. Marian, "The german experience with integrating photovoltaic systems into the low-voltage grids," Renewable energy, vol. 119, pp. 129-141, 2018.

[3] K. Divya and J. Østergaard, "Battery energy storage technology for power systemsan overview," Electric power systems research, vol. 79, no. 4, pp. 511-520, 2009.

[4] J. Moshövel, K.-P. Kairies, D. Magnor, M. Leuthold, M. Bost, S. Gährs, E. Szczechowicz, M. Cramer, and D. U. Sauer, "Analysis of the maximal possible grid relief from pv-peak-power impacts by using storage systems for increased self-consumption," Applied Energy, vol. 137, pp. 567-575, 2015.

[5] G. Angenendt, S. Zurmühlen, H. Axelsen, and D. U. Sauer, "Comparison of different operation strategies for pv battery home storage systems including forecast-based operation strategies," Applied energy, vol. 229, pp. 884-899, 2018.

[6] D. Maxwell, "Parts of Northern Ireland's electricity grid overloaded," https://www.bbc.com/news/uk-northern-ireland-24921411.

[7] M. Bost, B. Hirschl, and A. Aretz, Effekte von Eigenverbrauch und Netzparität bei der Photovoltaik. Institut für ökologische Wirtschaftsforschung (IÖW) $\mathrm{GmbH}$, gemeinnützig, 2011.

[8] J. Vetter, P. Novák, M. R. Wagner, C. Veit, K.-C. Möller, J. Besenhard, M. Winter, M. Wohlfahrt-Mehrens, C. Vogler, and A. Hammouche, "Ageing mechanisms in lithium-ion batteries," Journal of power sources, vol. 147 , no. 1-2, pp. 269-281, 2005.

[9] J. Li and M. A. Danzer, "Optimal charge control strategies for stationary photovoltaic battery systems," Journal of Power Sources, vol. 258, pp. 365-373, 2014.

[10] M. Schneider, P. Boras, H. Schaede, L. Quurck, and S. Rinderknecht, "Effects of operational strategies on performance and costs of electric energy storage systems," Energy Procedia, vol. 46, pp. 271-280, 2014.

[11] M. Trifkovic, M. Sheikhzadeh, K. Nigim, and P. Daoutidis, "Modeling and control of a renewable hybrid energy system with hydrogen storage," IEEE Transactions on Control Systems Technology, vol. 22, no. 1, pp. $169-179,2013$

[12] U. Raveendran Nair and R. Costa-Castelló, "A model predictive controlbased energy management scheme for hybrid storage system in islanded microgrids," IEEE Access, vol. 8, pp. 97 809-97 822, 2020.

[13] A. Parisio, E. Rikos, and L. Glielmo, "A model predictive control approach to microgrid operation optimization," IEEE Transactions on Control Systems Technology, vol. 22, no. 5, pp. 1813-1827, 2014.

[14] M. Petrollese, L. Valverde, D. Cocco, G. Cau, and J. Guerra, "Realtime integration of optimal generation scheduling with MPC for the energy management of a renewable hydrogen-based microgrid," Applied Energy, vol. 166, pp. 96-106, 2016.

[15] F. Garcia-Torres and C. Bordons, "Optimal economical schedule of hydrogen-based microgrids with hybrid storage using model predictive control," IEEE Transactions on Industrial Electronics, vol. 62, no. 8, pp. 5195-5207, 2015.

[16] J. Ramos-Teodoro, F. Rodríguez, M. Berenguel, and J. L. Torres, "Heterogeneous resource management in energy hubs with self-consumption: Contributions and application example," Applied energy, vol. 229, pp. 537-550, 2018.

[17] Y. Guo, Q. Wu, H. Gao, X. Chen, J. Østergaard, and H. Xin, "Mpcbased coordinated voltage regulation for distribution networks with distributed generation and energy storage system," IEEE Transactions on Sustainable Energy, vol. 10, no. 4, pp. 1731-1739, 2018.

[18] Y. Du, J. Wu, S. Li, C. Long, and S. Onori, "Coordinated energy dispatch of autonomous microgrids with distributed mpc optimization," IEEE Transactions on Industrial Informatics, vol. 15, no. 9, pp. 5289-5298, 2019.

[19] U. Raveendran Nair, M. Sandelic, A. Sangwongwanich, T. Dragicevic, R. Costa Castelló, and F. Blaabjerg, "Grid congestion mitigation and battery degradation minimisation using model predictive control in pvbased microgrid," IEEE Transactions on Energy Conversion, pp. 1-1, 2020.

[20] B. Kouvaritakis and M. Cannon, "Model predictive control," Switzerland: Springer International Publishing, 2016. 
[21] S. Haykin, Neural networks: a comprehensive foundation. Prentice Hall PTR, 1994

[22] S. I. Vagropoulos, G. Chouliaras, E. G. Kardakos, C. K. Simoglou, and A. G. Bakirtzis, "Comparison of sarimax, sarima, modified sarima and ann-based models for short-term pv generation forecasting," in 2016 IEEE International Energy Conference (ENERGYCON). IEEE, 2016, pp. $1-6$.

[23] M. Abdel-Nasser and K. Mahmoud, "Accurate photovoltaic power forecasting models using deep 1stm-rnn," Neural Computing and Applications, vol. 31, no. 7, pp. 2727-2740, 2019.

[24] V. Nair and G. E. Hinton, "Rectified linear units improve restricted boltzmann machines," in Proceedings of the 27th international conference on machine learning (ICML-10), 2010, pp. 807-814.

[25] S. S. Haykin et al., Neural networks and learning machines/Simon Haykin. New York: Prentice Hall,, 2009.

[26] J. M. Maciejowski, Predictive control: with constraints. Pearson education, 2002

[27] J. W. Chinneck, "Practical optimization: a gentle introduction," Systems and Computer Engineering), Carleton University, Ottawa. http://www. sce. carleton. calfaculty/chinneck/po. html, 2006.

[28] K. S. Ng, C.-S. Moo, Y.-P. Chen, and Y.-C. Hsieh, "Enhanced coulomb counting method for estimating state-of-charge and state-of-health of lithium-ion batteries," Applied energy, vol. 86, no. 9, pp. 1506-1511, 2009.

[29] A. Barré, B. Deguilhem, S. Grolleau, M. Gérard, F. Suard, and D. Riu, "A review on lithium-ion battery ageing mechanisms and estimations for automotive applications," Journal of Power Sources, vol. 241, pp. 680-689, 2013

[30] E. C. Kerrigan and J. M. Maciejowski, "Soft constraints and exact penalty functions in model predictive control," 2000.

[31] L. Gurobi Optimization, "Gurobi optimizer reference manual," 2019. [Online]. Available: http://www.gurobi.com

[32] A. Richards and J. How, "Robust stable model predictive control with constraint tightening," in 2006 American Control Conference. IEEE, 2006, pp. 6-pp.

[33] D. L. Marino, K. Amarasinghe, and M. Manic, "Building energy load forecasting using deep neural networks," in IECON 2016-42nd Annual Conference of the IEEE Industrial Electronics Society. IEEE, 2016, pp. 7046-7051.

[34] T. Stetz, F. Marten, and M. Braun, "Improved low voltage gridintegration of photovoltaic systems in germany," IEEE Transactions on sustainable energy, vol. 4, no. 2, pp. 534-542, 2012.

[35] M. Resch, B. Ramadhani, J. Bühler, and A. Sumper, "Comparison of control strategies of residential pv storage systems," in Proceedings 9th International Renewable Energy Storage Conference and Exhibition (IRES 2015), Messe Düsseldorf, 9-11 March 2015, 2015, pp. 1-18.

[36] F. Chollet et al., "Keras," https://keras.io, 2015.

[37] D. P. Kingma and J. Ba, "Adam: A method for stochastic optimization," arXiv preprint arXiv:1412.6980, 2014.

[38] L. Prechelt, "Automatic early stopping using cross validation: quantifying the criteria," Neural Networks, vol. 11, no. 4, pp. 761-767, 1998.

[39] B. Zakeri and S. Syri, "Electrical energy storage systems: A comparative life cycle cost analysis," Renewable and sustainable energy reviews, vol. 42, pp. 569-596, 2015 